\title{
Autochthonous acute hepatitis E: treatment with sofosbuvir and ribavirin
}

\author{
Elisa Biliotti ${ }^{1}$. Cristiana Franchi ${ }^{1}$. Martina Spaziante ${ }^{1} \cdot$ Anna Rosa Garbuglia ${ }^{2} \cdot$ Lorenzo Volpicelli $^{1}$. \\ Donatella Palazzo $^{1} \cdot$ Maurizio De Angelis ${ }^{1} \cdot$ Rozenn Esvan $^{1} \cdot$ Gloria Taliani $^{1}$
}

Received: 16 February 2018 / Accepted: 19 June 2018 / Published online: 26 June 2018

○) Springer-Verlag GmbH Germany, part of Springer Nature 2018

\begin{abstract}
Introduction Hepatitis E virus (HEV) is an emerging cause of autochthonous-acute-hepatitis and acute-on-chronic-liverfailure in western countries. Treatment is not routinely used, despite ribavirin has a good antiviral effect. In vitro sofosbuvir inhibits HEV replication, but clinical data are lacking.

Case report We report a case of acute-on-chronic-liver-failure due to HEV treated with sofosbuvir and ribavirin. The treatment was capable of rapidly inducing both HCV and HEV viral suppression.

Conclusion In conclusion, although more data are required before firm conclusions could be drawn, the combination of sofosbuvir and ribavirin in not immunosuppressed patients with acute hepatitis E may be able to clear HEV infection.
\end{abstract}

Keywords Hepatitis E · Ribavirin · Sofosbuvir · Acute-on-chronic-liver-failure $\cdot$ Hepatitis C

\section{Introduction}

Hepatitis E virus (HEV) infection is an emerging cause of autochthonous sporadic acute hepatitis in western countries [1]. The most common route of transmission is the manipulation or consumption of contaminated meat [2]. The infection is usually self-limiting; however a high mortality has been reported in patients developing acute-on-chronicliver-failure [3]. In such patients antiviral treatment is not routinely used, despite several reports demonstrating good antiviral effect of ribavirin [1,4]. A recent in vitro study showed that sofosbuvir is able to inhibit replication of HEV, with ribavirin exerting additive role in viral suppression [5]. However, clinical data concerning the HEV antiviral activity of sofosbuvir are currently lacking. We report the case of an acute-on-chronic-liver-failure due to HEV infection treated with sofosbuvir and ribavirin.

Elisa Biliotti

elisabiliotti@yahoo.it; elisa.biliotti@uniroma1.it

1 Department of Clinical Medicine, Clinic of Tropical Medicine, Sapienza University of Rome, Rome, Italy

2 Laboratory of Virology, National Institute for Infectious Diseases “Lazzaro Spallanzani”, Rome, Italy

\section{Case report}

A 57-year-old man with hepatitis $\mathrm{C}$ cirrhosis (HCV genotype 3) was diagnosed with an acute-on-chronic-liver-failure due to an acute HEV infection. He referred occasional consumption of uncooked wild boar sausages. His medical history included diabetes mellitus treated with metformin $500 \mathrm{mg}$ three times daily and arterial hypertension treated with ramipril $10 \mathrm{mg}$ once daily. At hospital admission, on March 1, 2016, he complained fever, malaise, nausea, abdominal pain and diarrhea with hypocholic stools lasting 1 week. Physical examination revealed jaundice, hepatic encephalopathy and ascites. Laboratory evaluations showed ALT $650 \mathrm{U} / \mathrm{L}$, bilirubin $33 \mathrm{mg} / \mathrm{dL}$, albumin $2.9 \mathrm{~g} / \mathrm{dL}$, INR 2.1, e-GFR $44 \mathrm{~mL} /$ $\mathrm{min} / 1.73 \mathrm{~m}^{2}$. Child-Pugh score was C-11 and Meld-Na score was 38. Both hepatitis viruses showed low replicating levels: HCV-RNA (VERSANT ${ }^{\circledR}$ HCV RNA 1.0 Assay) was 803 IU/ $\mathrm{mL}$ and HEV-RNA (Real-Star HEV RT-PCR Kit, Altona Diagnostics, Hamburg, GmbH) was 2285 copies/mL. HEV genotype was $3 \mathrm{e}$ and it was typed by sequencing an ORF2 fragment, as previously described [6]. After evaluation for liver transplantation, on March 4, treatment with sofosbuvir $(400 \mathrm{mg} /$ daily) and ribavirin $(800 \mathrm{mg} /$ daily $)$ was started. On March 9, both HCV-RNA and HEV-RNA were undetectable, ALT dropped to $207 \mathrm{U} / \mathrm{L}$, total bilirubin was $30 \mathrm{mg} /$ $\mathrm{dl}$, the Meld-Na score was 32 and the clinical conditions 
significantly improved along with regression of hepatic encephalopathy. By March 23, ALT value was 48 U/L, bilirubin was $23 \mathrm{mg} / \mathrm{dL}$, Meld-Na was 28 and both viruses were still undetectable (Fig. 1).

Unfortunately, the clinical course was complicated by the occurrence of sepsis due to methicillin-resistant Staphylococcus aureus (MRSA) and on April 4 the patient died of septic shock. On post-mortem examination, HEV-RNA (HEV ORF-1 and ORF-2) was undetectable by real-time PCR in liver, cardiac and muscle tissues.

\section{Conclusions}

Our clinical observation suggests that the combination of sofosbuvir and ribavirin in a patient with acute on chronic HEV infection was capable of rapidly inducing viral suppression. It is difficult to define the role of sofosbuvir in clearing HEV-RNA, since it was simultaneously administered with ribavirin. However, the largest published study on ribavirin treatment of acute hepatitis $\mathrm{E}$ indicated that the median time to HEV-RNA clearance was 29 days [1], while in our case it was only 5 days, suggesting that sofosbuvir had an effective additive role. We cannot exclude that the clearance of HEV-RNA from plasma may be due to spontaneous elimination, but the close temporal relationship between treatment administration and viral clearance allows us to suppose that sofosbuvir and ribavirin played an important role. Therefore, our clinical observation supports previous in vitro data, which indicate that the combination of sofosbuvir and ribavirin was able to inhibit HEV replication [5].

In three recently published cases, treatment with sofosbuvir was questioned for its partial effect on viral replication, being unable to clear HEV infection [7-9]. However, a few issues should be considered. First, the three reported patients were immunocompromised: one was immunosuppressed with tacrolimus and azathioprine post-liver-transplantation [7], one had chronic-lymphaticleukemia (CLL) treated with allogenic stem cell transplantation and retreated with fludarabin/cyclophosphamide and rituximab for relapsed CLL [8] and one had HIV infection with a CD4+ cell count lower than 100/ $\mu$ L [9]. Second, all patients had chronic HEV infection. Third, one patient did not receive ribavirin in combination with sofosbuvir because of a previously diagnosed intolerance [7], in the other two patients a long-term ribavirin treatment had been administered before sofosbuvir therapy and induced the D1384N, K1383N and/or G1634R mutations, clinically associated with ribavirin-resistant phenotype and persistently detectable HEV-RNA [8, 9]. Therefore, sofosbuvir operated in the presence of a functionally ineffective ribavirin. In these three patients with chronic HEV infection and well-documented impaired $\mathrm{T}$ cell function, sofosbuvir was given as a single potential antiviral agent and resulted ineffective to control HEV. In addition, two of the patients had HEV-3c [7] and 3i infection [9], respectively, while the patient we describe had HEV-3e infection. It remains to be clarified whether different HEV genotype/subtype have different sensitivity to sofosbuvir.

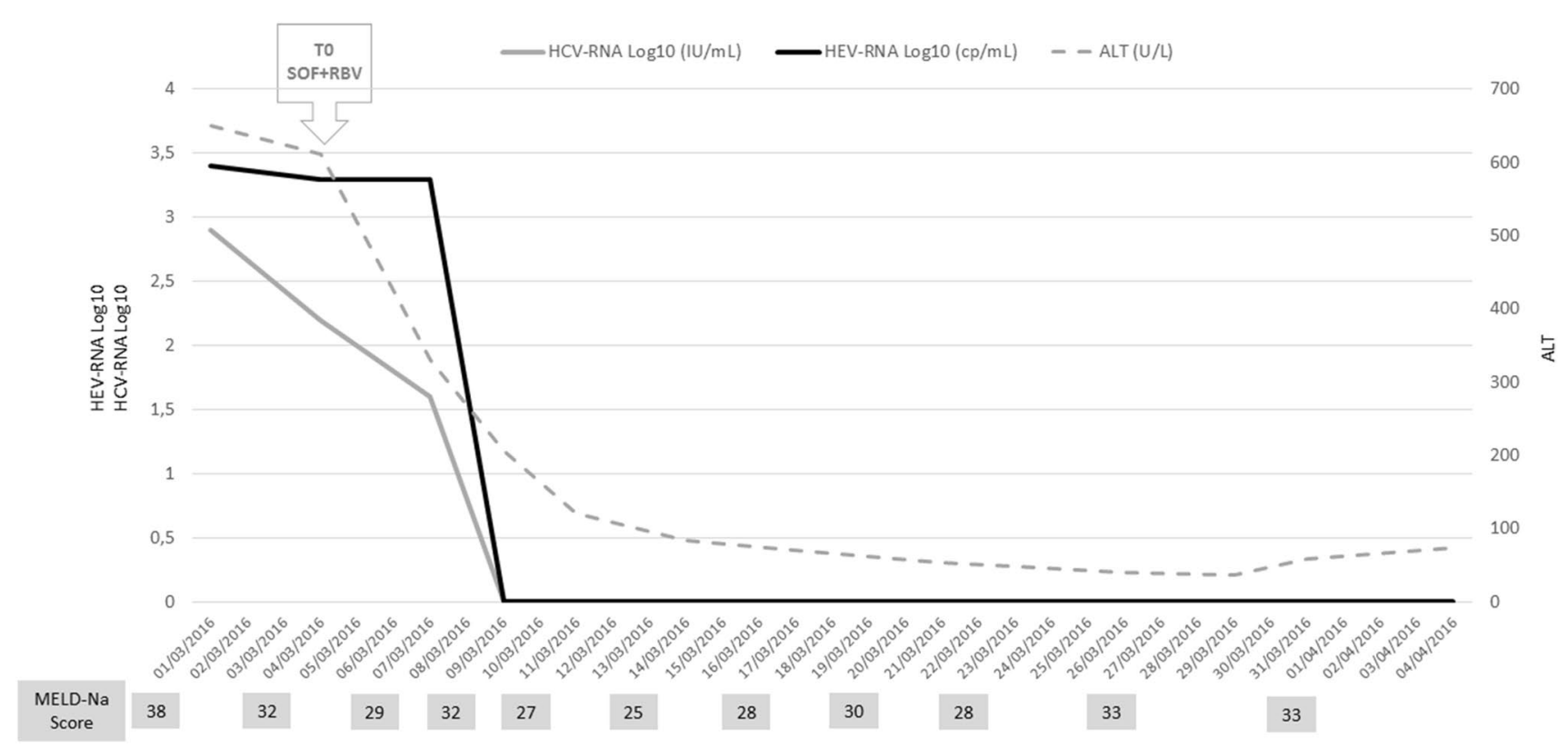

Fig. 1 Alanine aminotransferase (ALT). Hepatitis C virus viral load (HCV-RNA), hepatitis E virus viral load (HEV-RNA), Model for end-stage liver disease-sodium score (MELD-Na) during sofosbuvir (SOF) and ribavirin (RBV) treatment 
Finally, although we were unable to provide direct evidence, it seems reasonable that uncooked wild boar sausages were the source of HEV infection in this subject. In fact, the patient did not refer other risk factors such as travelling to endemic countries or blood transfusions and the infection was due to HEV genotype 3, which causes autochthonous HEV infection in developed countries and whose transmission is linked to the consumption of undercooked or raw organs from infected swine or wild boar [10]. This case draws attention to potential benefit of advising patients with underlying chronic liver diseases about the risk of acquiring HEV from undercooked meat, particularly processed pork or wild boar.

In conclusion, although more data are required before firm conclusions could be drawn, the virological outcome we observed indicates that the combination of sofosbuvir and ribavirin in not immunosuppressed patients with acute hepatitis E may be able to clear HEV infection.

\section{Compliance with ethical standards}

Conflict of interest All authors have nothing to disclose regarding funding or conflict of interest with respect to this manuscript.

\section{References}

1. Peron JM, Abravanel F, Guillaume M, Gérolami R, Nana J, Anty R, et al. Treatment of autochthonous acute hepatitis E with short-term ribavirin: a multicenter retrospective study. Liver Int. 2016;36:328-33.

2. Riveiro-Barciela M, Minguez B, Girones R, Rodriguez-Frías F, Quer J, Buti M. Phylogenetic demonstration of hepatitis E infection transmitted by pork meat ingestion. J Clin Gastroenterol. 2015;49:165-8.

3. Péron JM, Bureau C, Poirson H, Mansuy JM, Alric L, Selves J, et al. Fulminant liver failure from acute autochthonous hepatitis E in France: description of seven patients with acute hepatitis E and encephalopathy. J Viral Hepat. 2007;14:298-303.

4. Kamar N, Izopet J, Tripon S, Bismuth M, Hillaire S, Dumortier J, et al. Ribavirin for chronic hepatitis $\mathrm{E}$ virus infection in transplant recipients. N Engl J Med. 2014;370:1111-20.

5. Dao Thi VL, Debing Y, Wu X, Rice CM, Neyts J, Moradpour $\mathrm{D}$, et al. Sofosbuvir inhibits hepatitis $\mathrm{E}$ virus replication in vitro and results in an additive effect when combined with ribavirin. Gastroenterology. 2016;150:82-5.

6. Garbuglia AR, Scognamiglio P, Petrosillo N, Mastroianni CM, Sordillo P, Gentile D, et al. Hepatitis E virus genotype 4 outbreak, Italy, 2011. Emerg Infect Dis. 2013;19:110-4.

7. Donnelly MC, Imlach SN, Abravanel F, Ramalingam S, Johannessen I, Petrik J, et al. Sofosbuvir and daclatasvir anti-viral therapy fails to clear HEV viremia and restore reactive T cells in a HEV/ HCV co-infected liver transplant recipient. Gastroenterology. 2017;152:300-1.

8. Van der Valk M, Zaaijer HL, Kater AP, Schinkel J. Sofosbuvir shows antiviral activity in a patient with chronic hepatitis $\mathrm{E}$ virus infection. J Hepatol. 2017;66:242-3.

9. Todesco E, Demeret S, Calin R, Roque-Afonso AM, Thibault V, Mallet V, et al. Chronic hepatitis E in HIV/HBV coinfected patient: lack of power of sofosbuvir-ribavirin. AIDS. 2017;31:1346-8.

10. Pavio N, Doceul V, Bagdassarian E, Johne R. Recent knowledge on hepatitis E virus in Suidae reservoirs and transmission routes to human. Vet Res. 2017;48:78. 